\title{
Feature Extraction of Ship Radiation Signals Based on Wavelet Packet Decomposition and Energy Entropy
}

\author{
Yuxing Li $\mathbb{D},{ }^{1,2}$ Feiyue Ning $\mathbb{D},{ }^{1}$ Xinru Jiang $\mathbb{D},{ }^{1}$ and Yingmin $Y i \mathbb{D}^{1,2}$ \\ ${ }^{1}$ School of Automation and Information Engineering, Xi'an University of Technology, Xi'an 710048, China \\ ${ }^{2}$ Shaanxi Key Laboratory of Complex System Control and Intelligent Information Processing, Xi'an University of Technology, \\ Xi'an 710048, China \\ Correspondence should be addressed to Yingmin Yi; yiym@xaut.edu.cn
}

Received 12 September 2021; Revised 11 December 2021; Accepted 18 December 2021; Published 3 January 2022

Academic Editor: Akif Akgul

Copyright (C) 2022 Yuxing Li et al. This is an open access article distributed under the Creative Commons Attribution License, which permits unrestricted use, distribution, and reproduction in any medium, provided the original work is properly cited.

\begin{abstract}
The analysis of ship radiation signals to identify ships is an important research content of underwater acoustic signal processing. The traditional fast Fourier transform (FFT) is not suitable for analyzing non-stationary, non-Gaussian, and nonlinear signal processing. In order to realize the feature extraction and accurate classification of ship radiation signals with higher accuracy, a feature extraction method of ship radiation signals based on wavelet packet decomposition and energy entropy is proposed in this paper. According to wavelet packet decomposition, the ship radiation signal is decomposed into different frequency bands, and its energy entropy feature is extracted. As for comparisons, the center frequency and permutation entropy are also used as features to be extracted, then the $k$-nearest neighbor is applied to classify and recognize the extracted results. Based on the comparisons of wavelet packet decomposition, the center frequency, permutation entropy, and the $k$-nearest neighbor are used for classification and recognition. The experimental results present that, when comparing with center frequency and permutation entropy, the method based on energy entropy has the best availability, with the highest average recognition rate for four types of ship radiation signals, up to $98 \%$.
\end{abstract}

\section{Introduction}

In the development of science and technology, underwater acoustic signal processing plays an extremely important role in the human exploration of the ocean and plays a great role in military and marine life research $[1,2]$. Since the radiated noise signal of a ship contains a lot of information about the ship's characteristics, it has become one of the important indicators to measure the ship's performance, and it is widely used as the information source of passive sonar for the detection, tracking, and classification of ship targets $[3,4]$, so the feature extraction of the ship radiated noise signal has always been an important research content in the field of underwater acoustic signal processing, which has important theoretical and practical significance [5-8].

In general, many nonlinear, dynamic, and statistical parameters can be extracted as features of ship's radiated noise and for signal classification, such as the Lempel Ziv complexity (LZC) $[9,10]$, Lyapunov exponent [11], correlation coefficient analysis [12], spectral kurtosis [13, 14], and the entropy-based complexity, among which the entropybased complexity has attracted widespread attention. Entropy, as an indicator of the complexity of time series, can quantify the randomness of a time series, and greater entropy also represents greater irregularity $[15,16]$. Some commonly used entropies include approximate entropy [17], sample entropy [18], and permutation entropy [15]. Compared with approximate entropy and sample entropy, the arrangement is not easily affected by the length of the time series, and the calculation time is relatively short, so it has been applied in many fields [19-21]. However, the PE only considers the order of amplitude values and ignores some information of amplitude values. Therefore, the traditional PE algorithm should be improved [22].

Since the late 1980s, many scholars have also applied spectrum analysis to underwater acoustic target feature 
extraction, such as extracting the line spectrum and modulation spectrum of underwater target radiated noise by using acoustic power spectrum [23], Wigner-Ville distribution (WVD) [24], bispectrum and high-order spectrum analysis [25], empirical mode decomposition (EMD) [26], and wavelet or wavelet packet decomposition [27]. In particular, as a preprocessor, wavelet packet decomposition can divide the frequency space into various finite frequency bands to realize the time-frequency localization of the signal. It can decompose not only the low frequency but also the high frequency of the signal, and adaptively select the corresponding frequency band according to the analyzed signal, which has good timefrequency characteristics. Hence, wavelet packet decomposition is also widely used in signal feature extraction in various fields [28-30].

In this paper, we introduce the wavelet packet decomposition on the basis of energy entropy and calculate the corresponding PE value for each frequency band after decomposition and use it as a feature. Finally, the k-nearest neighbor $(\mathrm{KNN})$ algorithm is introduced and used as a classifier to verify the validity of the extracted features. A large number of comparative experimental results also prove the effectiveness of the feature extraction method proposed in this paper.

The train of thought of this paper is given as follows: Section 2 gives an introduction to wavelet packet decomposition; Section 3 proposes a feature extraction method based on PE and wavelet packet decomposition; Section 4 verifies the effectiveness of the feature extraction method proposed in this paper through comparative experiments; Section 5 embraces main conclusions obtained.

\section{Wavelet Packet Decomposition}

Wavelet packet decomposition, also known as an optimal sub-band tree structure, is a further optimization of the wavelet transform. The main algorithm idea is on the basis of the wavelet transform, in each stage of signal decomposition, not only the low-frequency sub-band is further decomposed, but also the high-frequency sub-band is further decomposed. The original signal is decomposed by the optimal signal decomposition path, which was calculated by minimizing a cost function.

$h_{k}$ and $g_{k}$ is the filter coefficient of the scale function, $g_{k}=(-1)^{k} h_{1-k}$ The wavelet function is equivalent to highpass filtering the signal to preserve the change details, while the scaling function is equivalent to low-pass filtering the signal to preserve the smooth shape. The basic function of the wavelet is actually formed by scaling and translating the scale function and the wavelet function. The size of the shift is related to the current scaling degree.

The scaling function $\psi(x)$ and wavelet function $\varphi(x)$ satisfy the following equations:

$$
\begin{aligned}
& \phi(x)=\sqrt{2} \sum_{k} h_{k} \phi(2 x-k), \\
& \psi(x)=\sqrt{2} \sum_{k} g_{k} \phi(2 x-k) .
\end{aligned}
$$

The time-domain signal $\mu(x)$ can be decomposed recursively as

$$
\begin{aligned}
\mu_{j, 2 n}(x) & =\sum_{k} h_{k} \mu_{j-1, n}(2 x-k), \\
\mu_{j, 2 n+1}(x) & =\sum_{k} g_{k} \mu_{j-1, n}(2 x-k),
\end{aligned}
$$

where $n=0,1, \ldots, 2(j-1)-1, g_{k}, h_{k}$ is low pass filter and high pass filter, respectively. When $n=0, \mu_{0,0}(x)=\varphi(x)$ $\mu_{0,1}(x)=\psi(x), \mu_{j, n}(x)$ denotes the wavelet coefficients at the $j^{\text {th }}$ level, $n^{\text {th }}$ frequency band. The recursive function set $\left\{\mu_{n}(x)\right\}_{n \in Z}$ defined in the abovementioned formula is the wavelet packet defined by the orthogonal scaling function. Figure 1 shows an example of a three-layer wavelet packet decomposition of signals.

From Figure 1, the wavelet packet of the signal is decomposed into three layers into eight frequency bands, and each layer is decomposed into two parts, high-frequency and low-frequency. The high-frequency and low-frequency coefficients of wavelet packet coefficients are decomposed again, unlike the wavelet decomposition, which only decomposes low-frequency coefficients.

\section{Proposed Feature Extraction Method}

Let $P=\left\{P_{1}, P_{2}, \ldots, P_{n}\right\}$ be a probability distribution, its information entropy $H(P)$ can be expressed as

$$
H(P)=\sum_{i=1}^{n} P_{i} \ln P_{i} .
$$

The difference of signal in time-frequency distribution can be expressed by the uncertainty of energy distribution in different frequency bands. Therefore, a feature extraction method of ship radiated signal based on wavelet packet decomposition and energy entropy is proposed in this paper. The wavelet packet is used to decompose ship signals to obtain different frequency bands. Energy entropy, as a feature, is used to estimate the uncertainty of ship signal frequency band energy distribution. The specific steps of the proposed feature extraction method are as follows:

(1) The ship signal is decomposed into $M$ frequency bands by wavelet packet decomposition. In this paper, a three-layer wavelet packet decomposition is performed, where the $M$ value is 8 The sum of the energy of the $M$ frequency bands is equal to the total energy of the ship signal.

(2) The energy proportion of each frequency band signal is calculated by 


$$
C_{i}=\frac{E_{i}}{E}
$$

$E_{i}$ is the energy of the $i^{\text {th }}$ frequency band signal; $E$ is the total energy of ship signal; $C_{i}$ is the proportion of the $i^{\text {th }}$ band signal to the total energy of the ship signal.

The total energy of the ship signal can be expressed as

$$
E=\sum_{i=1}^{M} E_{i}
$$

$M$ is the number of signals in different frequency bands obtained after wavelet packet decomposition.

(3) The energy entropy of the ship signal after wavelet packet decomposition is calculated by the definition of information entropy, which can be defined as

$$
H(C)=-\sum_{i=1}^{M} C_{i} \ln C_{i}
$$

(4) To demonstrate the effectiveness of the proposed feature extraction method, the $k$-nearest neighbor algorithm is used in the classification of the energy entropy features of ship signals to obtain the identification results. Given a training data set, for a new input instance, $\mathrm{K}$ instances closest to the instance are found in the training data set. Most of the $\mathrm{K}$ instances belong to a class, and the input instance is classified into this class.

\section{Feature Extraction of Ship Radiated Noise}

4.1. Ship Radiation Signals. In this paper, feature extraction and classification identification are carried out for four types of measured ship radiation signals (SRS), named SRS1, SRS2, SRS3, and SRS4. The signal lengths of the first and second types are 1380000 , the third type is 28282835 , and the fourth type is 164160 , and the sampling frequency is $44.1 \mathrm{kHz}$. Data points within 5001 to 1000000 of the four types of SRS are taken, Figure 2 presents four types of SRS after normalization.

4.2. Wavelet Packet Decomposition of Ship Radiation Signal. For the wavelet packet decomposition of four types of SRS, 100 samples are extracted from each type of SRS, each sample contains 5000 sampling points. One sample for each type of SRS is processed with the wavelet packet decomposition, using the DB2 wavelet basis function, each sample is decomposed into eight frequency bands (FB), named FB1, FB2, FB3, FB4, FB5, FB6, FB7, and FB8. The wavelet packet decomposition results of four types of SRS are shown in Figure 3 ( 1 sample). For the four types of SRS, the amplitudes in different FB have obvious differences, FB1 has the lowest frequency.
4.3. Feature Extraction Experiment. The energy entropy feature of each FB is extracted, center frequency (CF) and permutation entropy (PE) are used as comparative features.

4.3.1. Energy Entropy. Four types of SRS are extracted from the perspective of energy, the energy proportion is calculated for each FB. Figure 4 displays the energy distribution of four types of SRS (1 sample).

From Figure 4, for the four types of SRS, FB1 has the highest energy; the energy of SRS1 and SRS4 in FB2 is greater than that of SRS2 and SRS3; for FB2 of the four types of SRS, SRS4 has the most energy distribution, and similarly, for FB3 and FB4, SRS4 is also the highest; in the eight FB, the energy distributions of SRS2 are almost all concentrated in FB1, while those of SRS4 are more dispersed. Figure 5 exhibits the energy entropy of 100 samples for four types of ship signals.

In the light of Figure 5, there is a big difference in energy entropy among the four types of SRS; SRS2 has the smallest range of sample distribution, and has great stability; most samples of SRS1 are distributed between 0.4 and 0.7 , while a small number of samples of SRS3 and SRS4 also appears in this range; there is a clear order of the energy entropy of the samples for the four kinds of SRS.

4.3.2. Center Frequency. The CF feature extraction is performed for each FB of the four types of SRS, and the CF of each FB for the four types of SRS is shown in Figure 6.

As presented in Figure 6, for the four types of SRS, the CF under the same FB is different; for SRS2, the CF of FB7 and 8 is very different from the other three types of SRS; the CF distribution of samples in FB4 and FB6 is similar, the CF of SRS1 and SRS2 are close, and so are SRS3 and SRS4, but there is an obvious gap between the two groups; the $\mathrm{CF}$ distribution of the samples for SRS1 and SRS3 are very dispersed in each FB.

4.3.3. Permutation Entropy. PE mainly includes three parameters: data length, embedding dimension, and delay time, which are 5000, 3, and 1, respectively, in this experiment. Figure 7 indicates the PE of each FB for the four types of SRS.

As exhibited in Figure 7, the PE for the four types of SRS in the same FB is very close; the PE of the SRS2 coincides less with the range of PE distributions from the other SRS in FB1 and 7; SRS3 has a difference in its PE from the other SRS in FB5; the distribution of PE of samples for each type of SRS in each FB is very scattered.

\subsection{Analysis of Classification and Recognition Results}

4.4.1. K-Nearest Neighbor. $\mathrm{KNN}$ is one of the machine learning methods that classify each record in a data collection. The principle is that if a majority of the $\mathrm{K}$ most similar samples in a feature space belong to a class, then that sample also belongs to that class. This method is simple and easy to understand and implement, and $\mathrm{K}$ is set to 5 in this 


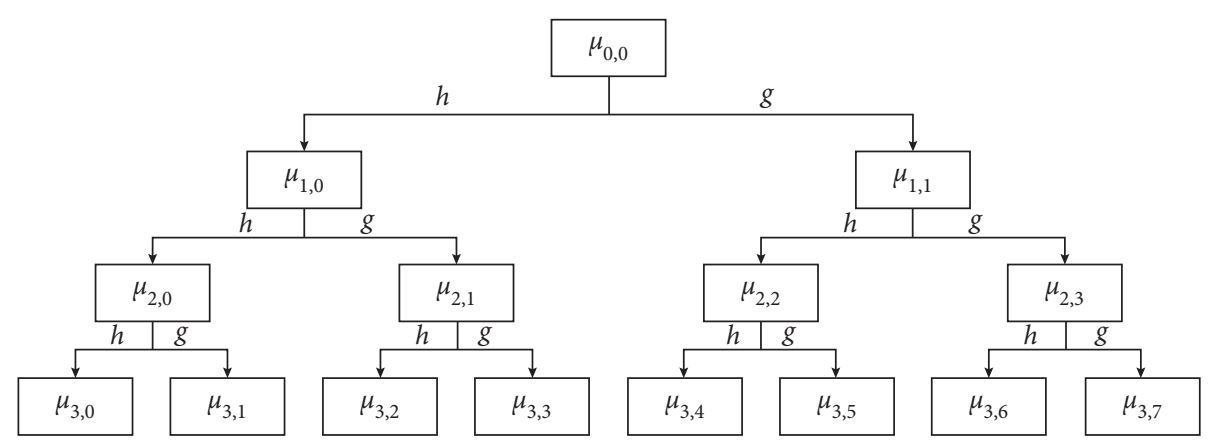

FIgURE 1: Three-layer wavelet packet decomposition.

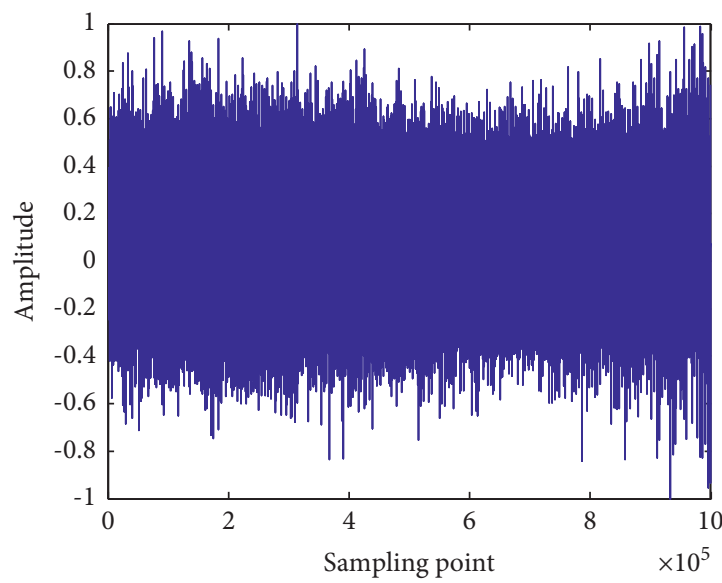

(a)

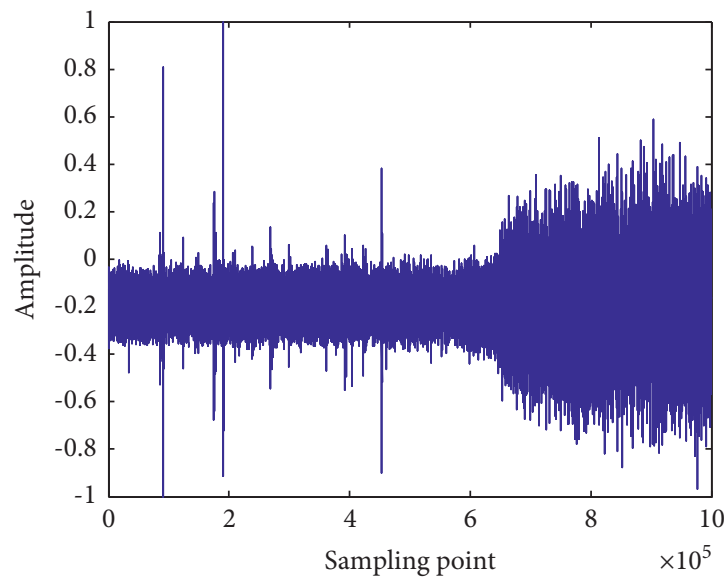

(c)

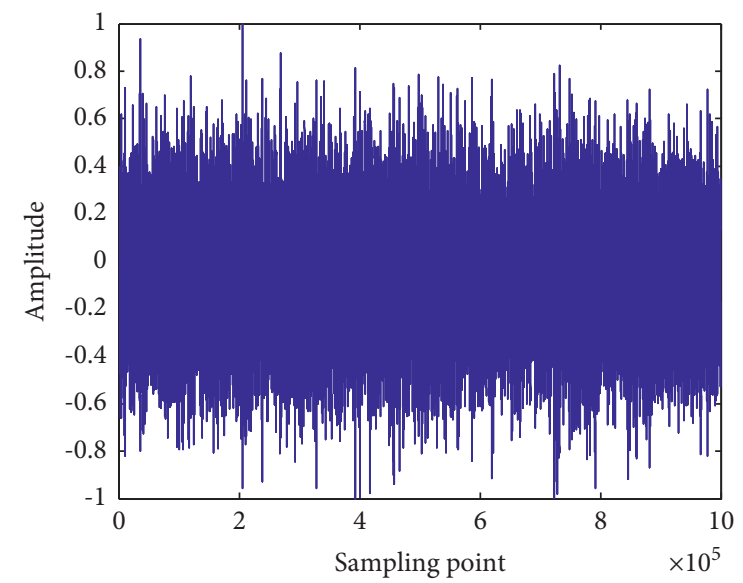

(b)

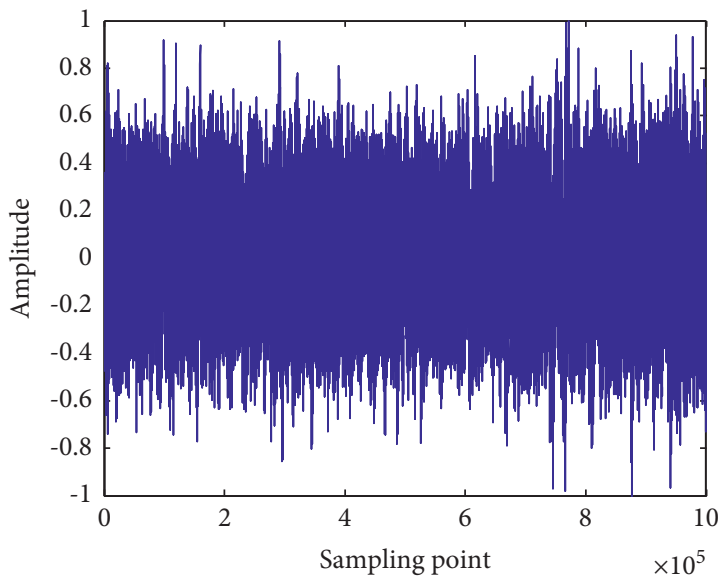

(d)

FIgURE 2: Four types of SRS after normalization. (a) SRS1. (b) SRS2. (c) SRS3. (d) SRS4.

paper. 50 samples of each type of SRS are selected as the training set and the remaining 50 as the test samples to classify and identify the four types of SRS. The recognition rate of each feature extraction method is obtained to compare and prove the effectiveness of the proposed method. The recognition rates of the energy entropy for four types of SRS are presented in Table 1.

According to Table 1, the recognition rate for each type of SRS is greater than $90 \%$, the recognition rates of SRS1 and
SRS2 are $100 \%$; the average recognition rate is $98 \%$; it is indicated that all the four types of SRS have good recognition results. The recognition rates of CF for four types of SRS are demonstrated in Table 2.

From Table 2, for the CF of each FB, the highest recognition rates of SRS1 and SRS4 is only 74\%, the recognition rate of SRS2 reaches 100\%; for SRS3, the recognition rates of each FB have a great difference; the highest average recognition rate of the four SRS is only $72 \%$, which is difficult to 


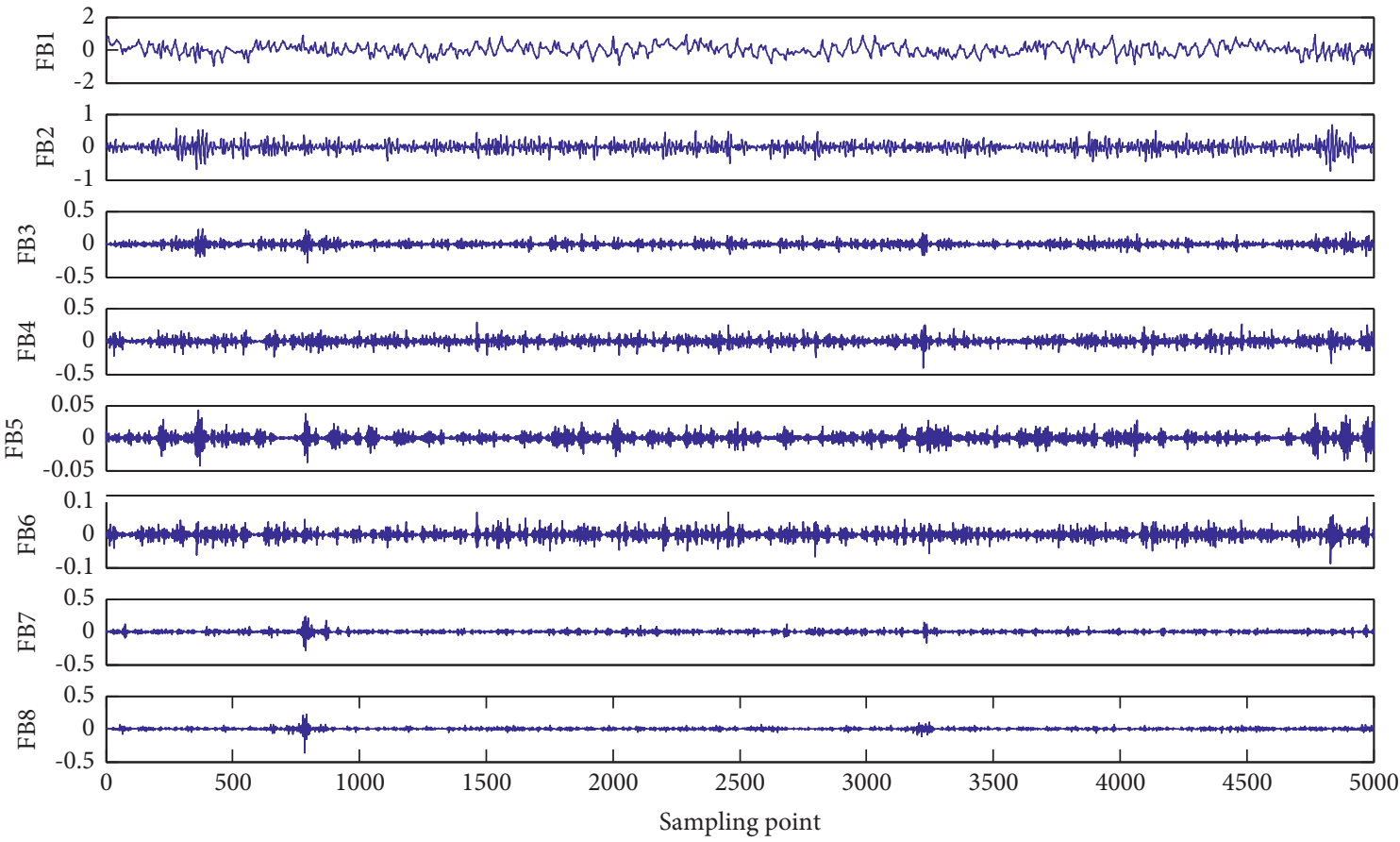

(a)

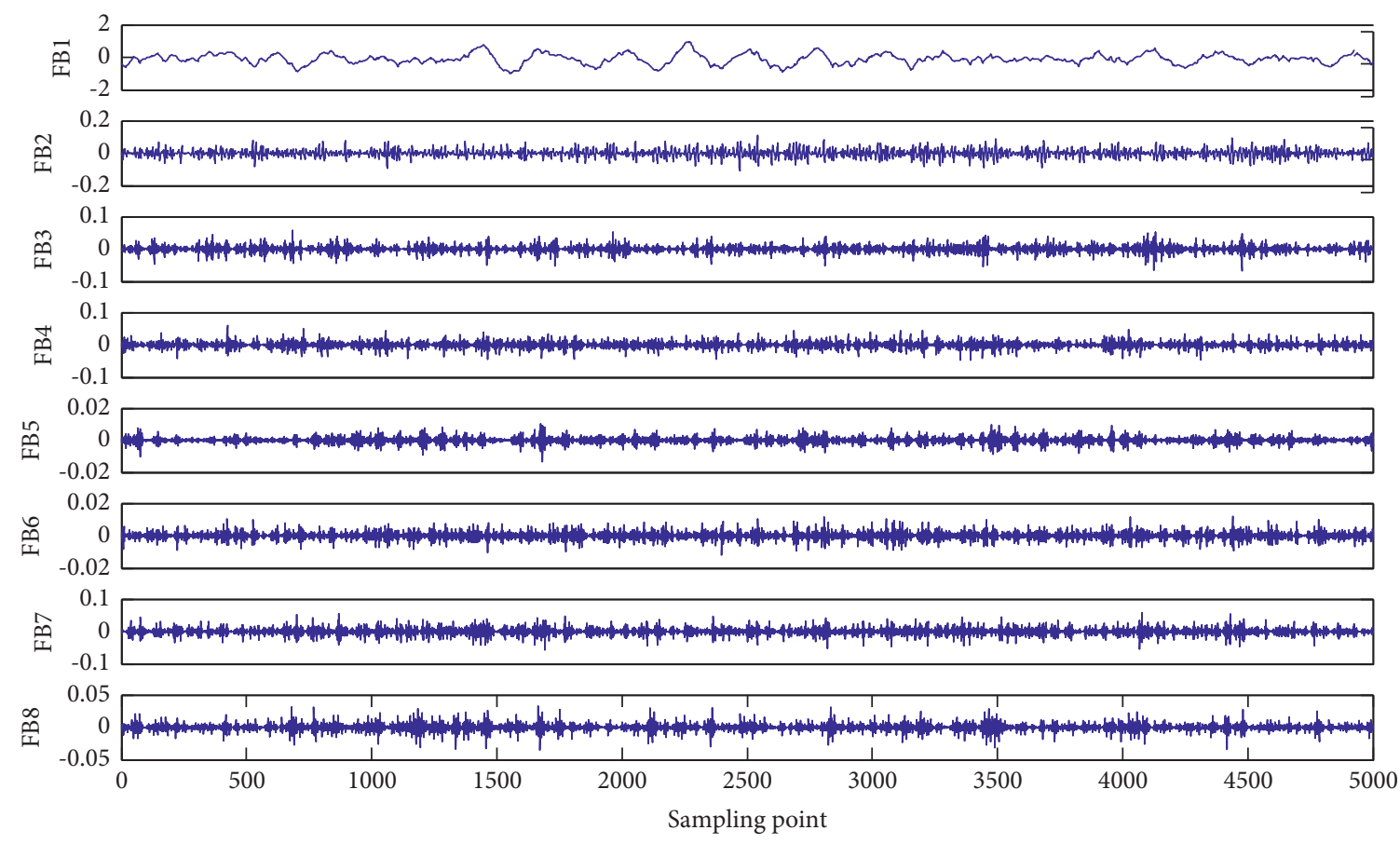

(b)

Figure 3: Continued. 


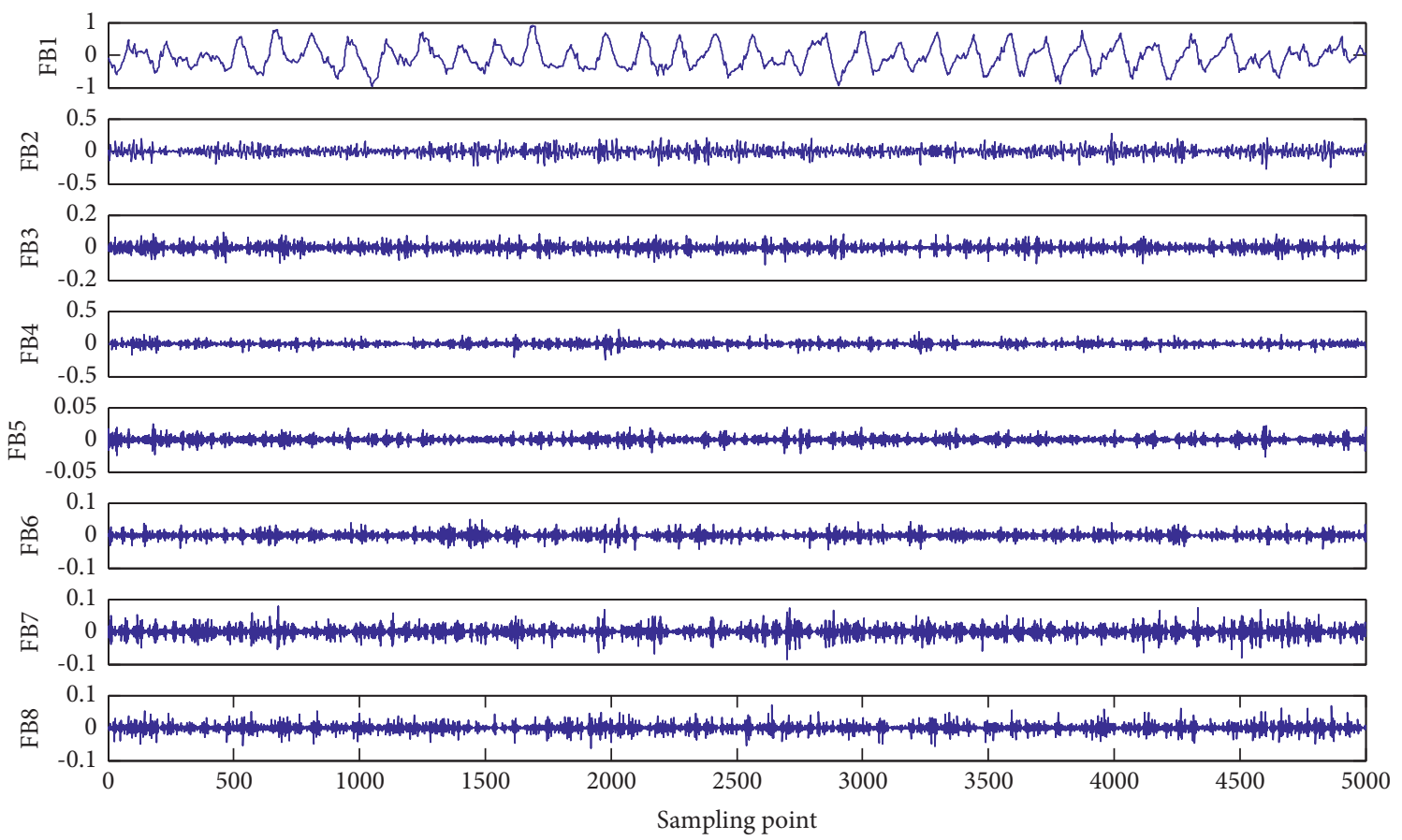

(c)

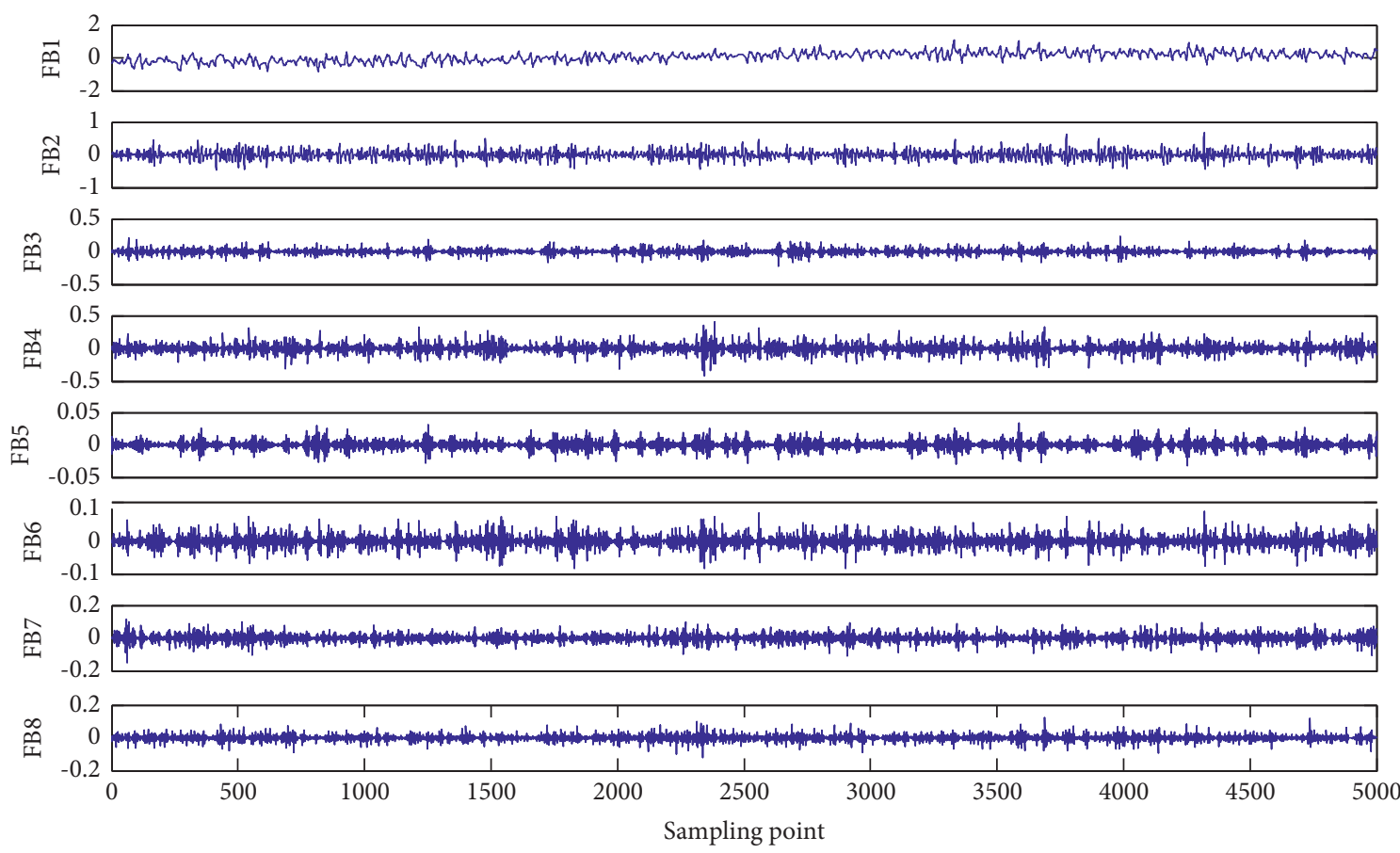

(d)

Figure 3: Wavelet packet decomposition results of four types of SRS (1 sample). (a) SRS1. (b) SRS2. (c) SRS3. (d) SRS4.

distinguish between the four SRS. The recognition rates of PE of four types of SRS are displayed in Table 3.

As can be seen from Table 3, overall, only FB6 had the highest average recognition rate of $52 \%$, and the other $\mathrm{FB}$ average recognition rates are below 50\%; the recognition rates of PE for SRS1 is less than $60 \%$, for SRS3 and SRS4 is less than $70 \%$; only the highest recognition rate of SRS2 is $86 \%$; the recognition results make it hard to classify and 


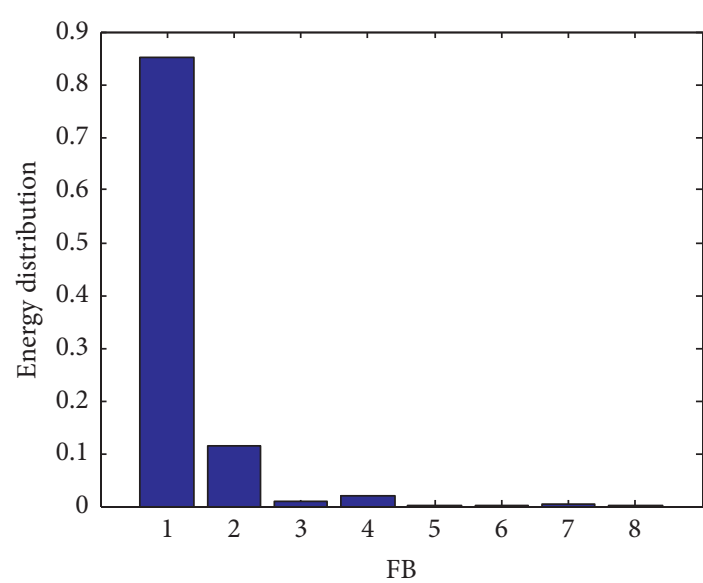

(a)

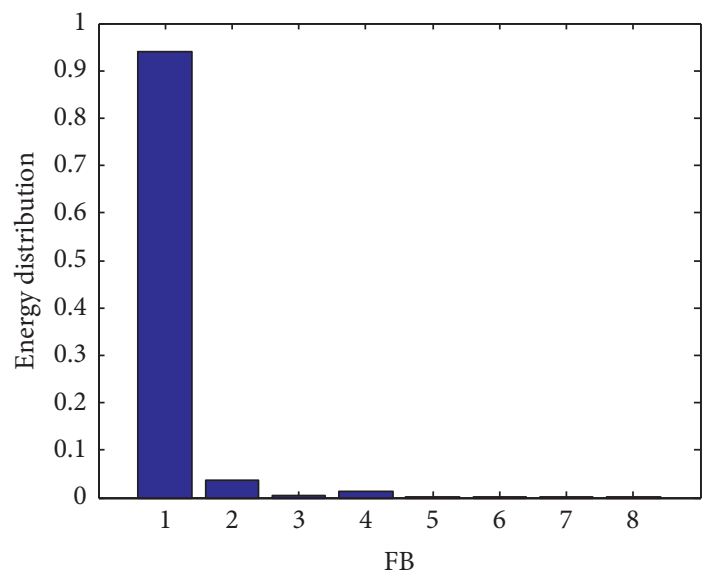

(c)

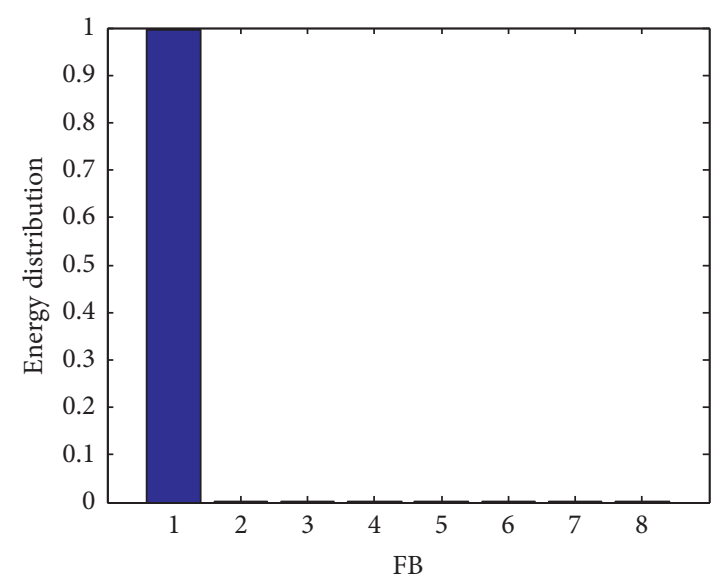

(b)

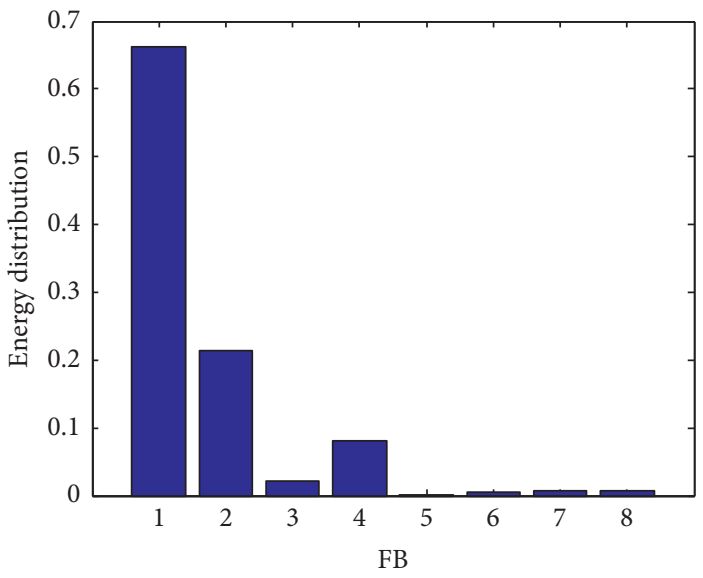

(d)

FIgURE 4: Energy distribution of four types of SRS (1 sample). (a) SRS1. (b) SRS2. (c) SRS3. (d) SRS4.

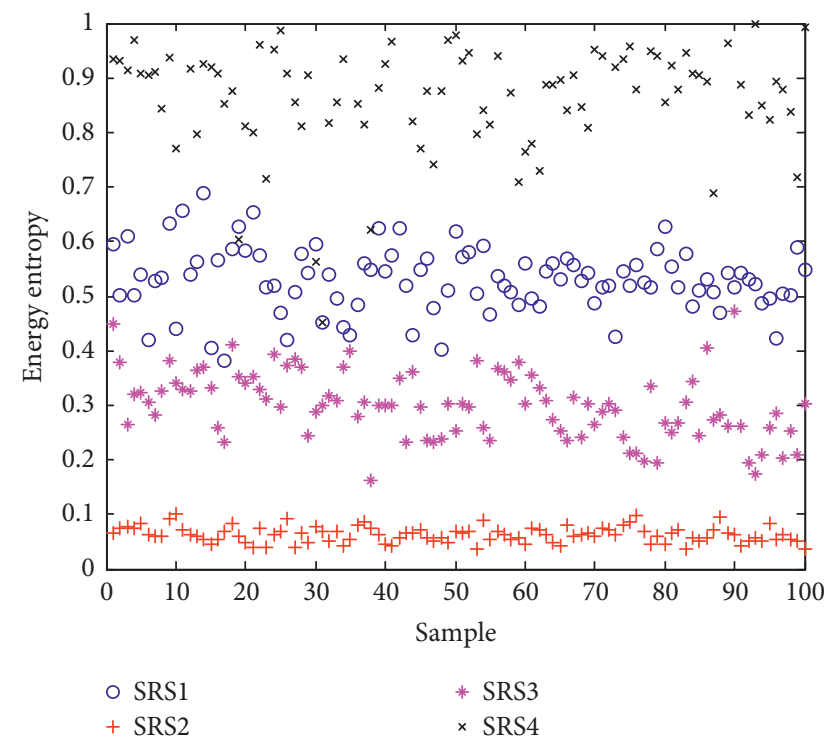

FIGURE 5: Energy entropy of 100 samples for four types of SRS. recognize the four types of SRS as well. For comparison, the highest recognition rates of the three methods are displayed in Table 4.

It is known from Table 4 that the feature extraction based on wavelet packet decomposition and energy entropy can effectively identify four types of SRS with the highest recognition rate of $98 \%$, much better than the other two.

4.4.2. Random Forest. In this subsection, the random forest (RF) method is introduced to compare with $\mathrm{KNN}, \mathrm{RF}$ is a machine learning method that integrates multiple trees by the idea of ensemble learning. Its basic unit is the decision tree, and its essence is an important branch of machine learning - ensemble learning method and the number of trees in this paper is set to 25. As in Section 4.4.1, 50 training samples and 50 test samples for each type of SRS are taken. The recognition rates of the energy 7entropy for four types of SRS are presented in Table 5.

Table 5 shows that the recognition rates of both SRS1 and SRS2 reached $100 \%$; for each type of SRS, the recognition 


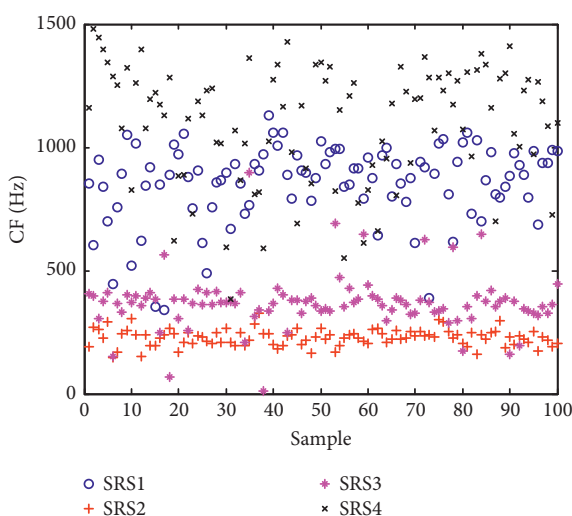

(a)

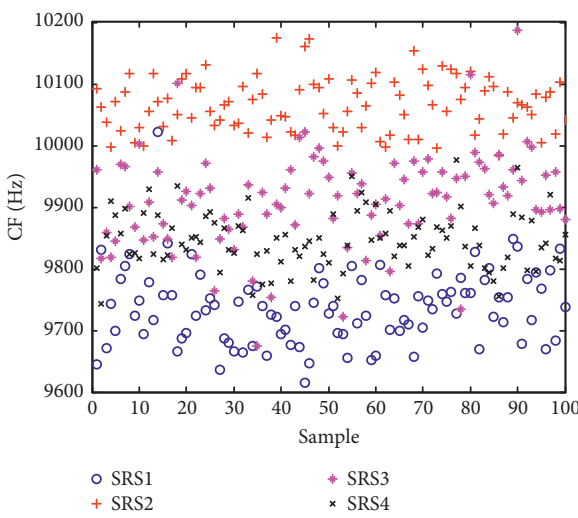

(c)

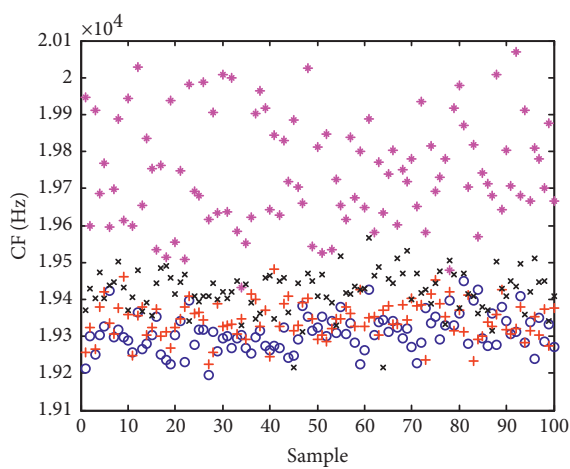

- SRS1

* SRS3
$\times$ SRS4

(e)

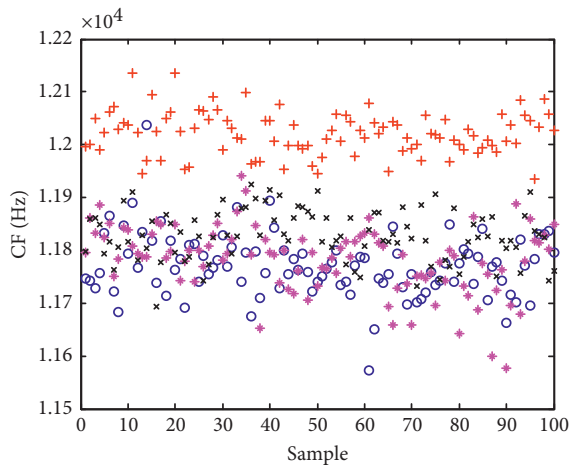

- SRS1

* SRS3

(g)

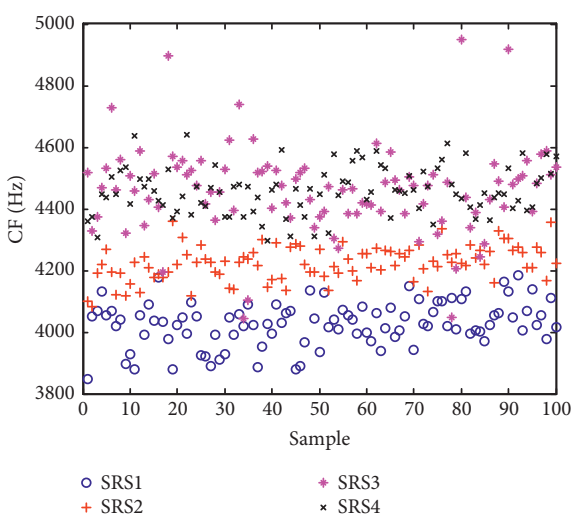

(b)

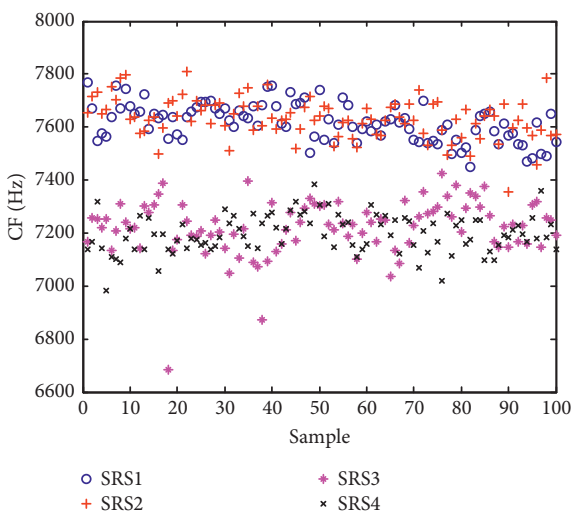

(d)

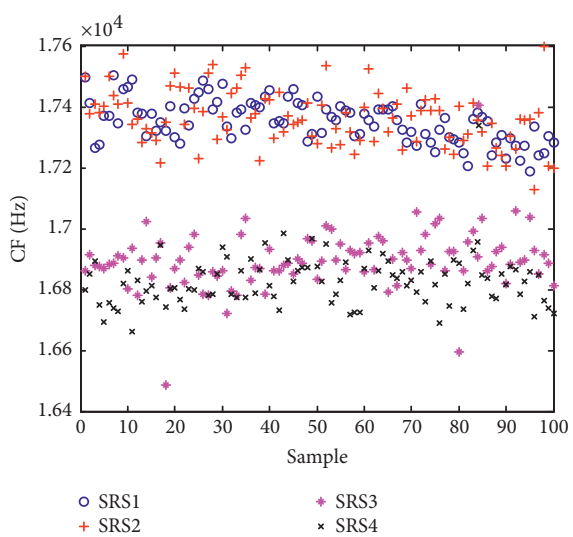

(f)

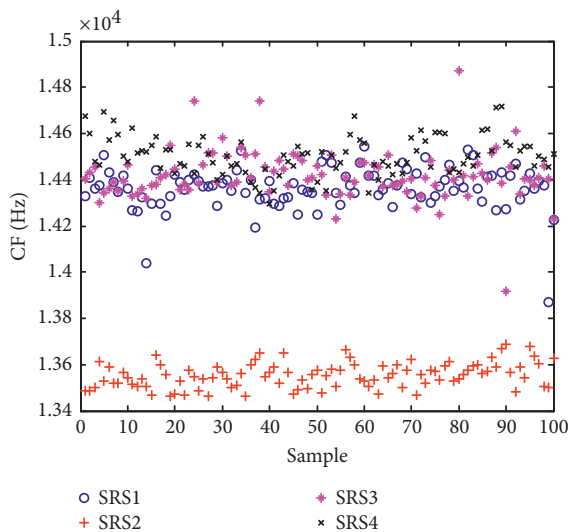

(h)

Figure 6: CF of each FB for the four types of SRS. (a) FB1. (b) FB2. (c) FB3. (d) FB4. (e) FB5. (f) FB6. (g) FB7. (h) FB8. 


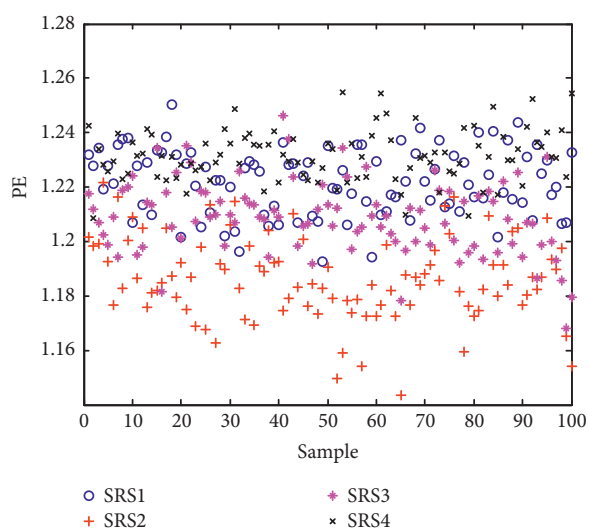

(a)

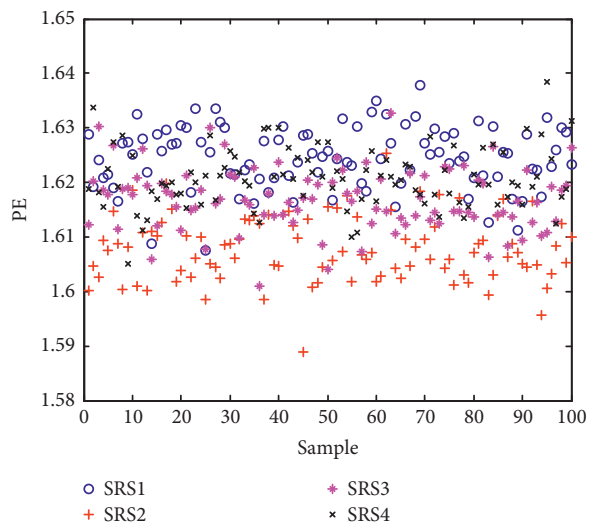

(c)

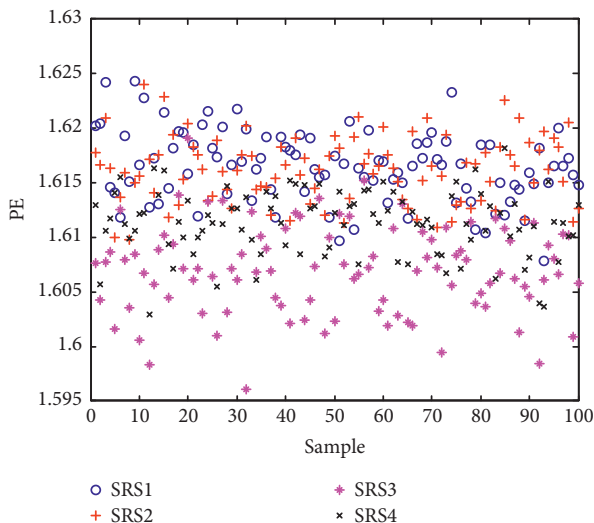

(e)

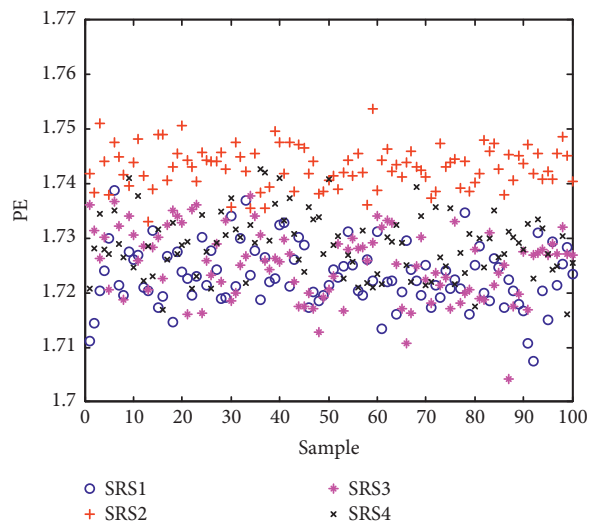

(g)

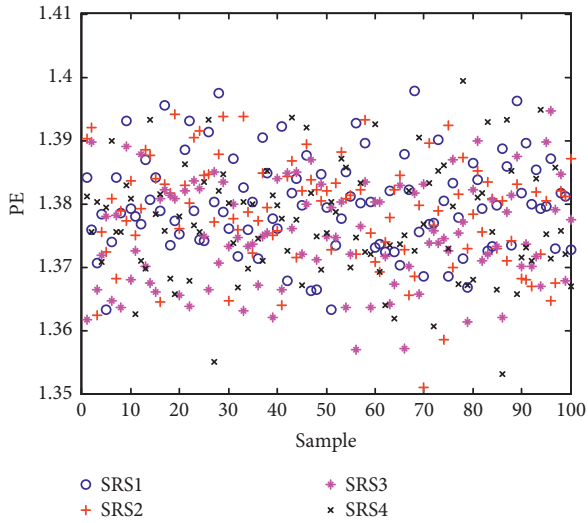

(b)

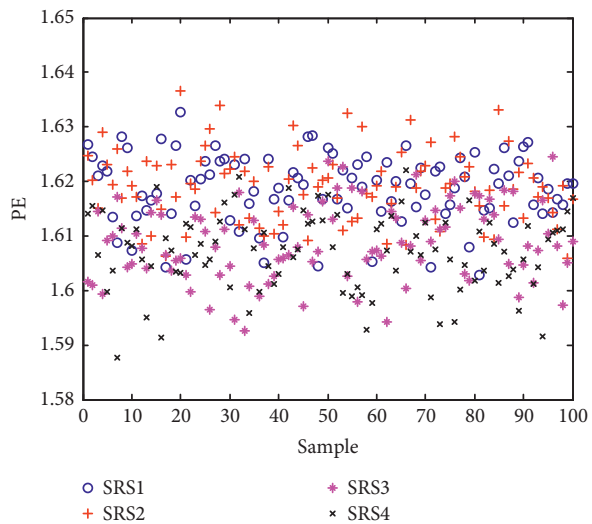

(d)

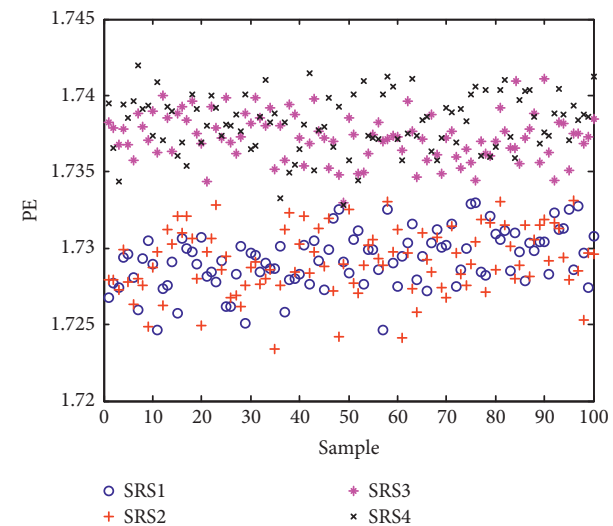

(f)

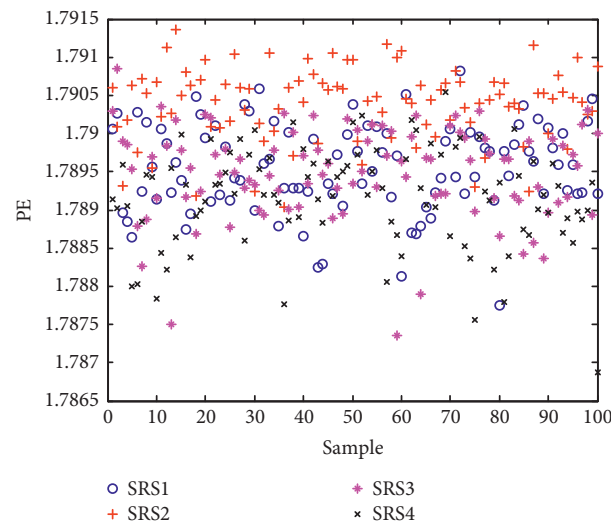

(h)

Figure 7: PE of each FB for the four types of SRS. (a) FB1. (b) FB2. (c) FB3. (d) FB4. (e) FB5. (f) FB6. (g) FB7. (h) FB8. 
TABLE 1: The recognition rates of energy entropy for four types of SRS.

\begin{tabular}{lcccc}
\hline SRS1 & SRS2 & SRS3 & SRS4 & Average \\
$100 \%$ & $100 \%$ & $94 \%$ & $98 \%$ & $98 \%$ \\
\hline
\end{tabular}

TABLE 2: The recognition rates of CF for four types of SRS.

\begin{tabular}{lccccc}
\hline & SRS1 (\%) & SRS2 (\%) & SRS3 (\%) & SRS4 (\%) & Average (\%) \\
\hline FB1 & 62 & 98 & 54 & 74 & 72 \\
FB2 & 74 & 88 & 36 & 38 & 59 \\
FB3 & 66 & 90 & 64 & 42 & 65.5 \\
FB4 & 52 & 56 & 40 & 52 & 50 \\
FB5 & 48 & 44 & 98 & 68 & 64.5 \\
FB6 & 48 & 56 & 72 & 56 & 58 \\
FB7 & 34 & 98 & 26 & 48 & 51.5 \\
FB8 & 40 & 100 & 42 & 66 & 62 \\
\hline
\end{tabular}

TABLE 3: The recognition rates of PE for four types of SRS.

\begin{tabular}{lccccc}
\hline & SRS1 (\%) & SRS2 (\%) & SRS3 (\%) & SRS4 (\%) & Average (\%) \\
\hline FB1 & 20 & 80 & 42 & 46 & 47 \\
FB2 & 30 & 20 & 38 & 36 & 31 \\
FB3 & 56 & 64 & 22 & 32 & 43.5 \\
FB4 & 26 & 46 & 38 & 34 & 36 \\
FB5 & 34 & 32 & 64 & 40 & 42.5 \\
FB6 & 38 & 42 & 60 & 68 & 52 \\
FB7 & 32 & 86 & 42 & 36 & 49 \\
FB8 & 24 & 60 & 20 & 34 & 34.5 \\
\hline
\end{tabular}

TABLE 4: The highest recognition rates of the three feature extraction methods.

\begin{tabular}{lcc}
\hline Energy entropy & $\mathrm{CF}$ & $\mathrm{PE}$ \\
$98 \%$ & $72 \%$ & $52 \%$ \\
\hline
\end{tabular}

TABLE 5: The recognition rates of energy entropy for four types of SRS (Random forests).

\begin{tabular}{llccc}
\hline SRS1 & SRS2 & SRS3 & SRS4 & Average \\
$100 \%$ & $100 \%$ & $94 \%$ & $96 \%$ & $97.5 \%$ \\
\hline
\end{tabular}

TABLE 6: The recognition rates of CF for four types of SRS (Random forests).

\begin{tabular}{lccccc}
\hline & SRS1 (\%) & SRS2 (\%) & SRS3 (\%) & SRS4 (\%) & Average (\%) \\
\hline FB1 & 72 & 98 & 42 & 76 & 72 \\
FB2 & 74 & 88 & 36 & 32 & 57.5 \\
FB3 & 72 & 92 & 68 & 36 & 67 \\
FB4 & 58 & 46 & 56 & 48 & 52 \\
FB5 & 50 & 40 & 98 & 60 & 62 \\
FB6 & 54 & 52 & 84 & 38 & 57 \\
FB7 & 46 & 98 & 16 & 50 & 52.5 \\
FB8 & 50 & 100 & 38 & 52 & 60 \\
\hline
\end{tabular}

rates were generally higher than $90 \%$, and the average recognition rate reached $97.5 \%$, which verified the effectiveness of the feature extraction method proposed in this paper.

The recognition rates of $\mathrm{CF}$ for four types of SRS are displayed in Table 6 . From Table 6 , it can be seen that the highest average recognition rate of the four SRSs is $72 \%$ and the lowest is only $52 \%$; there is a significant difference in the recognition rate of each FB of SRS3; in FB7, the recognition rate of SRS2 is $98 \%$, but the recognition rate of SRS3 is only $16 \%$; these results imply that it is difficult to distinguish one SRS from another.

Table 7 shows the recognition rate of PE for the four types of SRSs, from Table 7, the recognition rate of PE is very low, none of the results has a recognition rate over $50 \%$; the highest recognition rate for SRS3 is $48 \%$; for SRS1 and SRS4, the recognition rate is below $40 \%$; the highest average recognition rate is $38.5 \%$, which indicates that the classification of the four types of SRSs by PE of random forest is very difficult.

The highest recognition rates of the three methods are shown in Table 8. As we can see from Table 8, with the recognition rate of $97.5 \%$, the energy entropy can recognize the SRS better than the other two features. The above results show that the influence of the classifier is not dominant, and the energy entropy-based feature extraction method proposed in this paper still has the highest recognition rate compared with the $\mathrm{CF}$ and $\mathrm{PE}$ in the comparison experiments with RF as the classifier.

\section{Discussion}

In this paper, energy entropy, $\mathrm{CF}$ and $\mathrm{PE}$ are used to extract the features for each FB of SRS. Energy entropy has a very significant effect in identifying the SRS. The CF is presented as a method other than entropy. The PE can easily and accurately locate the time when the system changes abruptly, and it can amplify the small changes of the signal. PE and energy entropy are in the form of entropy, so the results of permutation entropy and energy entropy are compared in this paper to prove the effectiveness of energy entropy. From the comparison results of recognition rates, we can see that energy entropy has better separability as an extracted feature compared to PE.

In the classification comparison experiment, we calculate the recognition rate while introducing different classifiers for comparison. For energy entropy, the recognition result of $\mathrm{KNN}$ is $98 \%$, while the recognition result of random forest is $97.5 \%$. Both classifiers prove the effectiveness of the feature extraction method proposed in this paper, but the result of $\mathrm{KNN}$ is better than RF, while the recognition results of CF and $\mathrm{PE}$ also prove this point.

Finally, we introduce cross-validation to avoid overfitting. Different numbers of training samples are randomly selected from 100 samples, and the rest are used as test samples. To reduce randomness, the cycle is repeated 20 times to compare whether the standard deviations are close under different numbers of training samples. The classification results of the proposed feature extraction method using a different number of samples for training are shown in Table 9.

As can be seen from the table, the average recognition rate at the end is similar for different training sample numbers, and the standard deviation fluctuates just a little, indicating that there is no overfitting problem. We can also 
TABLE 7: The recognition rates of PE for four types of SRS (Random forests).

\begin{tabular}{|c|c|c|c|c|c|}
\hline & SRS1 (\%) & SRS2 (\%) & SRS3 (\%) & SRS4 (\%) & Average (\%) \\
\hline FB1 & 26 & 48 & 40 & 28 & 35.5 \\
\hline FB2 & 32 & 28 & 24 & 20 & 26 \\
\hline FB3 & 20 & 34 & 20 & 20 & 23.5 \\
\hline FB4 & 20 & 34 & 32 & 30 & 29 \\
\hline FB5 & 30 & 28 & 36 & 30 & 31 \\
\hline FB6 & 36 & 38 & 48 & 32 & 38.5 \\
\hline FB7 & 34 & 46 & 34 & 30 & 36 \\
\hline FB8 & 16 & 24 & 18 & 28 & 21.5 \\
\hline
\end{tabular}

TABLE 8: The highest recognition rates of the three feature extraction methods (Random forests).

\begin{tabular}{lcc}
\hline Energy entropy & CF & PE \\
$97.5 \%$ & $72 \%$ & $38.5 \%$ \\
\hline
\end{tabular}

TABLE 9: The classification results of the proposed feature extraction method using a different number of samples for training.

\begin{tabular}{lcccccc}
\hline Number of training samples & 80 & 70 & 60 & 50 & 40 & 30 \\
\hline Average accuracy & $95.125 \%$ & $96.009 \%$ & $95.000 \%$ & $95.400 \%$ & $95.625 \%$ & $95.553 \%$ \\
Standard deviation & 0.0205 & 0.01848 & 0.0163 & 0.0086 & 0.01025 & 0.00971 \\
\hline
\end{tabular}

observe that when we select 50 training samples, the recognition rate reaches $95.4 \%$ with the standard deviation of 0.0086 , which is the most stable group under each combination in Table 9. Considering the average recognition rate and standard deviation, we select 50 samples for training and the remaining 50 for testing, so as to verify the advantages of the feature extraction method proposed in this paper.

\section{Conclusions}

According to this paper, the wavelet packet decomposition is integrated with energy entropy to extract features of four types of SRS. By comparing with CE and PE, the effectiveness of EE is testified, and the major conclusions are summarized as follows:

(1) In view of the advantages of wavelet analysis theory in non-stationary signal analysis and processing, and based on this theory, this paper presents an analysis method of SRS by combining energy entropy.

(2) Observing the conditions of figures and contrasting the recognition rates, it can be found that, when compared with CF and PE, the energy entropy can reflect the energy distribution of each FB from a macro perspective, which is more valid.

(3) Compared with other feature extraction methods, the feature extraction method of wavelet packet decomposition and energy entropy proposed in this paper has the highest recognition rate of $98 \%$, at least $26 \%$ higher. As a result, the availability of the new method can be fully confirmed.

\section{Data Availability}

The data used to support the findings of this study are available from the corresponding author upon request.

\section{Conflicts of Interest}

The authors declare that they have no conflicts of interest.

\section{References}

[1] Y. Li, Y. Li, X. Chen, and J. Yu, "A novel feature extraction method for ship-radiated noise based on variational mode decomposition and multi-scale permutation Entropy," Entropy, vol. 19, no. 7, 2017.

[2] X. Guo, Y. Li, and K. Yang, "On the dynamics of ocean ambient noise: two decades later," Chaos, vol. 25, Article ID 103117, 2015.

[3] S. Wang and X. Zeng, "Robust underwater noise targets classification using auditory inspired time-frequency analysis," Applied Acoustics, vol. 78, no. 4, pp. 68-76, 2014.

[4] S. Jiao, B. Geng, Y. Li, Q. Zhang, and Q. Wang, "Fluctuation-based reverse dispersion entropy and its applications to signal classification," Applied Acoustics, vol. 175, no. 4, Article ID 107857, 2021.

[5] Y. Li, S. Jiao, and B. Geng, "A comparative study of four multiscale entropies combined with grey relational degree in classification of ship-radiated noise," Applied Acoustics, vol. 176, no. 4, Article ID 107865, 2021.

[6] A. K. S. Jardine, D. Lin, and D. Banjevic, "A review on machinery diagnostics and prognostics implementing condition-based maintenance," Mechanical Systems and Signal Processing, vol. 20, no. 7, pp. 1483-1510, 2006.

[7] Y. Zhang, X. Li, L. Gao, L. Wang, and L. Wen, "Imbalanced data fault diagnosis of rotating machinery using synthetic oversampling and feature learning," Journal of Manufacturing Systems, vol. 48, pp. 34-50, 2018.

[8] Y. Li, X. Chen, J. Yu, X. Yang, and H. Yang, "The data-driven optimization method and its application in feature extraction of ship-radiated noise with sample entropy," Energies, vol. 12, no. 3, p. 359, 2019.

[9] A. Lempel and J. Ziv, "On the complexity of finite sequences," IEEE Transactions on Information Theory, vol. 22, no. 1, pp. 75-81, 1976. 
[10] L. Cui, X. Gong, J. Zhang, and H. Wang, "Double-dictionary matching pursuit for fault extent evaluation of rolling bearing based on the Lempel-Ziv complexity," Journal of Sound and Vibration, vol. 385, pp. 372-388, 2016.

[11] A. Babloyantz and A. Destexhe, "Is the normal heart a periodic oscillator?" Biological Cybernetics, vol. 58, no. 3, pp. 203-211, 1988.

[12] Z. Zhang, A. Verma, and A. Kusiak, "Fault analysis and condition monitoring of the wind turbine gearbox," IEEE Transactions on Energy Conversion, vol. 27, no. 2, pp. 526-535, 2012.

[13] J. Antoni and R. B. Randall, "The spectral kurtosis: application to the vibratory surveillance and diagnostics of rotating machines," Mechanical Systems and Signal Processing, vol. 20, no. 2, pp. 308-331, 2006.

[14] N. Sawalhi, R. B. Randall, and H. Endo, "The enhancement of fault detection and diagnosis in rolling element bearings using minimum entropy deconvolution combined with spectral kurtosis," Mechanical Systems and Signal Processing, vol. 21, no. 6, pp. 2616-2633, 2007.

[15] C. Bandt and B. Pompe, "Permutation entropy: a natural complexity measure for time series," Physical Review Letters, vol. 88, Article ID 174102, 2012.

[16] C. Bandt, "Ordinal time series analysis," Ecological Modelling, vol. 182, no. 3-4, pp. 229-238, 2005.

[17] S. M. Pincus, "Approximate entropy as a measure of system complexity," Proceedings of the National Academy of Sciences, vol. 88, no. 6, pp. 2297-2301, 1991.

[18] J. S. Richman and J. R. Moorman, "Physiological time-series analysis using approximate entropy and sample entropy," American Journal of Physiology - Heart and Circulatory Physiology, vol. 278, no. 6, pp. H2039-H2049, 2000.

[19] R. Alcaraz and J. J. Rieta, "A review on sample entropy applications for the non-invasive analysis of atrial fibrillation electrocardiograms," Biomedical Signal Processing and Control, vol. 5, no. 1, pp. 1-14, 2010.

[20] E. Olofsen, J. W. Sleigh, and A. Dahan, "Permutation entropy of the electroencephalogram: a measure of anaesthetic drug effect," British Journal of Anaesthesia, vol. 101, no. 6, pp. $810-821,2008$.

[21] J. M. Yentes, N. Hunt, K. K. Schmid, J. P. Kaipust, D. McGrath, and N. Stergiou, "The appropriate use of approximate entropy and sample entropy with short data sets," Annals of Biomedical Engineering, vol. 41, no. 2, pp. 349-365, 2013.

[22] Y. Bai, Z. Liang, and X. Li, "A permutation lempel-ziv complexity measure for EEG analysis," Biomedical Signal Processing and Control, vol. 19, pp. 102-114, 2015.

[23] D. V. Ha, V. D. Nguyen, and Q. K. Nguyen, "Modeling of Doppler power spectrum for underwater acoustic channels," Journal of Communications and Networks, vol. 19, no. 3, pp. 270-281, 2017.

[24] E. Widjiati, E. B. Jatmiko, W. Wardhana et al., "Analysis of propeller cavitation-induced signal using neural network and wigner-ville distribution," in Proceedings of the 2012 Oceans, Yeosu, Korea (South), May 2012.

[25] J. Wang and Y. Pi, "SAR tomography imaging via higherorder spectrum analysis," Journal of Systems Engineering and Electronics, vol. 04, pp. 748-754, 2009.

[26] Y. Li, L. Wang, X. Li, and X. Yang, "A novel linear spectrum frequency feature extraction technique for warship radio noise based on complete ensemble empirical mode decomposition with adaptive noise, duffing chaotic oscillator, and weighted-permutation entropy," Entropy, vol. 21, no. 5, p. 507, 2019.

[27] I. Cohen, S. Raz, and D. Malah, "Orthonormal shift-invariant wavelet packet decomposition and representation," Signal Processing, vol. 57, no. 3, pp. 251-270, 1997.

[28] B.. Luk, Z. D. Jiang, K. P. Liu, and F. Tong, "Impact acoustic non-destructive evaluation in noisy environment based on wavelet packet decomposition," Lecture Notes in Engineering and Computer Science, vol. 2169, no. 1, pp. 1338-1341, 2008.

[29] I. Saha and R. V. Sagar, "Classification of the acoustic emissions generated during the tensile fracture process in steel fibre reinforced concrete using a waveform-based clustering method," Construction and Building Materials, vol. 294, Article ID 123541, 2021.

[30] R. P. J. Kumar, C. Akila, R. Mercy, and M. D. Lakshmi, "Texture classification using wavelet packet decomposition based on SGS \& MISS algorithm," Digital Image Processing, vol. 36 , no. 5 , pp. 57-59, 2010. 